\title{
Social work practice implications of upcoming mental health reforms
}

\author{
Genevieve Smith ${ }^{1}$ and Joanna Appleby ${ }^{2}$
}

The Labour-led Government has recently announced new healthcare reforms in Aotearoa New Zealand. The four changes are:

- The current 20 District Health Boards (DHBs) across the country will be replaced by one national organisation, Health New Zealand.

- A new Māori Health Authority will have the power to commission health services, monitor the state of Māori health and develop policy

- New Public Health Agency will be created

- Strengthened Ministry of Health will monitor performance and advise government

As a teaser before the announcement, the country was informed that the reforms would be about equity and access. We, the writers, are both Pākehā mental health social workers and we were eagerly awaiting news of the reforms. We have reflected on the current problems facing the mental health sector, speculated on how these may be addressed through system reform, and discussed our hopes for a better system.

Based on our experience working within and alongside different DHB mental health services in the country we identified four major sector problems. These are the increasing demand and severity of presenting problems, the associated shifting goalposts of accessibility to manage demand, service variability between regions and issues of staff workload, and training and retention. As social workers, we have seen the impact of these issues upon multistressed families seeking support and we hope for reforms to address these equity issues.

\section{Increased demand and severity}

The Ministry of Health (2021) has recently released the Office of the Director of Mental Health and Addiction Services Annual Report 2018 and 2019 under a cloud of controversy surrounding the timeframe and lack of transparency (Cooke, 2021). The statistics of increased mental health service demand reflect our experience as mental health social workers. Referral demand is unprecedented, due in part to the excellent efforts of health promoters to reduce mental health stigma and encourage people to seek help. We have seen a change in recent years in how families perceive mental health issues, with increased understanding of mental health and acceptance of service involvement. The result is increased demand for mental health services without the service infrastructure to support that.

Barnett and Bagshaw (2020) have described the persistent underinvestment in health services in Aotearoa New Zealand over many years due to the business culture that has been embedded within health systems. In mental health services, we have seen the business culture evident in the focus on efficiency and throughput. In response to increased service demand, many services have turned to brief intervention as a model of care. In order to access supports, families must be prepared to talk with multiple clinicians. For some this does not seem to be a problem and for others this can turn them off services completely or lead to them bouncing around different agencies, getting
${ }^{1}$ Registered Social Worker, Auckland New Zealand

${ }^{2}$ University of Auckland, New Zealand
AOTEAROA NEW ZEALAND SOCIAL WORK 33(2), 61-65.

CORRESPONDENCE TO: Joanna Appleby joanna.appleby@gmail.com 
what they can, telling their story multiple times, getting support, but without treatment of the core problem. We are particularly concerned about the cultural appropriateness of the brief intervention model. It seems to be an individualistic model with time constraints limiting the scope of the engagement phase and the time to work with whānau and wider systems. This approach may exacerbate existing mental health inequalities in Aotearoa New Zealand and does not seem to be a good fit for Māori and Pacific families.

As advocates for formulation-driven work, we would like to see not only easier access, but support that can see someone through a longer period of time in the context of a trusted and stable therapeutic relationship. The quality of the therapeutic relationship is particularly important for Māori whānau (McClintock et al., 2011; Pomare, 2015) and for those who have had negative experiences with multiple services (Munford \& Sanders, 2015). The therapist and service may need to take a considerably different and more culturally appropriate approach in order to build a positive therapeutic relationship with Māori whānau (Bennett et al., 2016).

Alongside the referral increase there is an increase in the severity of problems facing people, with the impacts of COVID, increases in family violence and significant financial struggles. This results in referrals accepted into service being heavily dominated by serious problems such as acute suicide risk.

\section{Accessibility}

Despite the increase in referrals, DHB mental health staffing numbers appear to have remained the same. Services may respond to this imbalance of demand and available resource by narrowing service eligibility criteria. As a result, only the most severe mental health presentations are accepted into DHB care. This is not aligned with the vision of Te Ara Oranga (Government Inquiry into Mental Health and Addiction, 2018) about providing services when needed or any preventative model of care. Instead, underfunded services are forced to defer or delay services until a person's problems are exacerbated to the point of crisis. Wait times have grown over the last three years in child and adolescent mental health services across the country, almost doubling in Canterbury to 59 days (Ministry of Health, 2021). This is a very long time in the life of someone with severe mental health issues, especially a young person, during which time the problems can worsen.

These accessibility issues are of concern, particularly for those people and families who are reluctant to seek help and are most in need of timely and responsive care. This may be the result of internalised mental health stigma or institutional mistrust from poor experiences with services. Many Māori have had negative experiences with health services due to racism (Graham \& MastersAwatere, 2020) that can become a double disadvantage when it results in reluctance to seek help when required. These problems stem from how services meet (or do not meet) the mental health needs of all of our communities. Accessibility and equity, the keywords from the announcement teaser, are critically important for the mental health sector.

\section{Service variability}

From our experience the postcode lottery, as Health Minister Andrew Little has described, is a very real phenomenon within mental health services. There are significant differences between DHBs. Our experience is primarily in child and adolescent mental health services. Effective interventions with families include Wraparound approaches, Dialectical Behaviour Therapy (DBT) and Parent-Child Interaction Therapy (PCIT), all of which are supported by local research (Cooney \& Ministry of Health, 2010; Shailer et al., 2017; Woodfield \& Cartwright, 2020). However, these services are not available in every DHB. For example, it is highly 
likely for a family to be involved in a DBT programme in one $\mathrm{DHB}$, and then move to another area of Aotearoa New Zealand where a DBT programme is not available.

It appears that the services provided depend on the current staff knowledge, training and resources. This is likely due to training issues, but we also connect this with staff turnover due to the workload issues. We have seen many mental health staff join the $\mathrm{DHB}$, receive training in appropriate interventions, find the unrelenting workload intolerable and then leave the public sector to work privately, taking that knowledge with them. In particular, we have seen a mass exodus of psychologists from the public service as they seek a better work environment working privately (Truebridge, 2021).

\section{Staffing issues}

In recognition of these issues, there have been efforts put into recruiting and training more mental health staff. For social workers, there is the New Entry to Specialist Practice (NESP) programme providing structured academic and clinical learning in their first year of mental health practice (Te Pou, 2021). There is current research under way about social workers' experiences of NESP to ensure the programme is meeting the needs of social workers new to mental health practice.

However, we see the staffing issues are primarily about retaining staff in mental health services. Many mental health staff are struggling with burnout in the face of everincreasing service demand. There is a steady stream of referrals and the people who get into services tend to have significant and multiple problems. As social workers, it is challenging to practise ecologically in this work environment with limited time to work with families and other support systems. Despite efforts to support clinicians, it can be dissatisfying to work with acutely unwell people within limited timeframes to engage in meaningful work that could address some of the root causes of distress-all the while knowing that people are queuing at the door to be seen. As a result, we see a high turnover of staff resulting in less experienced clinicians and less support for those new to the role.

\section{Health service reforms}

It was against the backdrop of these four issues of service demand, accessibility, variability and staffing issues that we awaited the health service reform announcement.

We are cautiously optimistic about the proposed changes. Having Health New Zealand replace the DHBs could be helpful in addressing issues of regional service variability. Currently, the $20 \mathrm{DHBs}$ are funded by the Ministry of Health to plan, purchase and provide health and disability services within defined geographical areas. They have autonomy as to how to meet the directives set by the Ministry. The creation of Health New Zealand could mean regulation of mental health service entry criteria and interventions available across the country. Ideally, this will also involve one integrated IT system across the country. It seems absurd that there are currently different IT systems between DHBs so that we cannot easily access clinical notes for people who transfer from another area.

However, we do not yet know how this will be operationalised. Goodyear-Smith and Ashton (2019) have described the seven major health system reforms between 1983 and 2000 in Aotearoa New Zealand, all of which have failed to address the persistent problems of access to care and inequitable health outcomes. We do not know what impact the organisational restructure will have on local practice decisions. While regulation will help with access, there also needs to be flexibility to be responsive to local need. This includes the specific mental health needs within rural areas and socioeconomically deprived areas. 
The four issues of service demand, accessibility, variability and staff retention are intertwined and underscored by a lack of funding. Service demand results in services being less accessible through shifting goal posts of entry criteria. This creates a stressful workplace where clinicians are predominantly working with people presenting with severe problems and high risk. The business model creates pressure to work with complex problems in time-limited ways, which has likely been a major factor in a high turnover of staff. This impacts on what particular interventions are available in each service as departing staff take their expertise with them. For these health reforms to achieve their goal of equity and access, there needs to be accompanying funding to support mental health services to meet the needs of people seeking support. Lower caseloads could be one way that we can work more relationally and responsively and with meaningful engagement with families and wider systems in culturally appropriate ways, all of which are aligned with both social work and recovery principles.

The current issues within the mental health sector impact more on Māori whānau. Institutional racism is a major cause for Māori to experience a number of health disparities and ultimately to die, on average, seven years younger than non-Māori (Kerr, 2021). The current health system in general is often experienced as hostile and alienating for Māori (Graham \& Masters-Awatere, 2020) and mental health services need to do better for Māori, especially regarding the use of the Mental Health Act (Baker, 2015; Elder \& Tapsell, 2013; Government Inquiry into Mental Health and Addictions, 2018). We are supportive of the establishment of a new Māori Health Authority especially if this means that mental health services can be delivered in more holistic and relational ways. We hope that this new dual authority approach may move us towards a more Tiriti-centred health system that ensures equitable access and outcomes. We were particularly pleased to see that Tā Mason
Durie has been appointed, with his vast experience in mental health.

The upcoming reforms provide an opportunity to address some of the longstanding issues in mental health, especially if it means an integrated network of mental health services across the country. Alongside this, there needs to be adequate funding so that mental health services can move from a business model to a recovery model. This could create a work environment where clinicians have the time to work holistically, to be able to build rapport at a pace that suits the person seeking support, and to have the capacity to work with the system surrounding people. Being resourced to provide intensive support at the outset may also avoid the revolving door in and out of mental health services for some people. We continue to hold hope for a better system for the people and communities we serve.

\section{References}

Baker, M. (2015). He Kai i nga Rangatira: He korero o nga whānau whaiora. Te Rau Matatini.

Barnett, P., \& Bagshaw, P. (2020). Neoliberalism: What it is, how it affects health and what to do about it. The New Zealand Medical Journal, 133(1512), 76-84.

Bennett, S. T., Flett, R. A., \& Babbage, D. R. (2016). Considerations for culturally responsive cognitivebehavioural therapy for Māori with depression. Journal of Pacific Rim Psychology, 10(8), 1-11. https://doi. org/10.1017/prp.2016.5

Cooke, H. (2021, April 4). "A lot of data and negative statistics": Inside the battle behind dramatic edits and huge delays to a Government mental health report. Stuff https://www.stuff.co.nz/national/politics/300267615/a-lotof-data-and-negative-statistics-inside-the-battle-behinddramatic-edits-and-huge-delays-to-a-governmentmental-health-report

Cooney, E., \& Ministry of Health. (2010). Feasibility of evaluating $D B T$ for self-harming adolescent: $A$ small randomised controlled trial. Te Pou o Te Whakaaro Nui: The National Centre of Mental Health Research and Workforce Development.

Elder, H., \& Tapsell, R. (2013). Māori and the Mental Health Act. In J. Dawson \& K. Gledhill (Eds.). New Zealand's Mental Health Act in practice. Victoria University Press.

Goodyear-Smith, F., \& Ashton, T. (2019). New Zealand health system: Universalism struggles with persisting inequities. The Lancet, 394 (10196), 432-442.

Government Inquiry into Mental Health and Addiction. (2018). He Ara Oranga: Report of the Government Inquiry into Mental Health and Addiction. www.mentalhealth.inquiry. govt.nz/inquiry-report/ 
Graham, R., \& Masters-Awatere, B. (2020). Experiences of Māori of Aotearoa New Zealand's public health system: A systematic review of two decades of published qualitative research. Australia and New Zealand Journal of Public Health, 44, 193-200. doi:10.1111/17536405.12971

Kerr, F. (2021, April 22). Self-determination at the heart of a new Māori health authority. Stuff. https://www.stuff.co.nz/ pou-tiaki/300282517/selfdetermination-at-the-heart-ofnew-mori-health-authority

McClintock, K., Moeke-Maxwell, T., \& Mellsop, G. (2011). Appropriate child and adolescent mental health service (CAMHS): Māori caregiver's perspectives. Pimatisiwin: $A$ Journal of Aboriginal and Indigenous Community Health, 9(2), 387-398.

Ministry of Health. (2021). Office of the Director of Mental Health and Addiction Services Annual Report 2018 and 2019. Office of the Director of Mental Health and Addiction Services Annual Report 2018 and 2019 | Ministry of Health NZ

Munford, R., \& Sanders, J. (2015). Components of effective social work practice in mental health for young people who are users of multiple services. Social Work in Mental Health, 13(5), 415-438. doi:10.1080/15332985. 2014.959239

Pomare, P. P. (2015). He kākano ahau i ruia mai i rangiātea: Engaging Māori in culturally-responsive child and adolescent mental health services [Unpublished doctoral thesis]. University of Auckland.

Shailer, J. L., Gammon, R. A., \& de Terte, I. (2017). An investigation of the fidelity to a wraparound process in New Zealand. New Zealand Journal of Psychology, 46(2), 87-98.

Te Pou. (2021). New entry to specialist practice: Allied mental health and addiction. https://www.tepou.co.nz/ training-development/grants/nesp-allied-mental-healthand-addiction

Truebridge, N. (2021, February 23). Ex-DHB psychologist claims chronic failings in mental health services. Radio New Zealand. Ex-DHB psychologist claims chronic failings in mental health services | RNZ

Woodfield, M. J., \& Cartwright, C. (2020). Parent-child interaction therapy from the parents' perspective. Journal of Child and Family Studies, 29(3), 632-647. doi:10.1007/s10826-019-01611-5 